\title{
Can Correlations Drive a Band Insulator Metallic?
}

\author{
Arti Garg, ${ }^{1}$ H. R. Krishnamurthy, ${ }^{2}$ and Mohit Randeria ${ }^{3}$ \\ ${ }^{1}$ Department of Theoretical Physics, Tata Institute of Fundamental Research, Mumbai 400005, India \\ ${ }^{2}$ Centre for Condensed Matter Theory, Department of Physics, Indian Institute of Science, Bangalore 560012 , \\ and Condensed Matter Theory Unit, JNCASR, Jakkur, Bangalore 560 064, India \\ ${ }^{3}$ Department of Physics, The Ohio State University, Columbus, Ohio 43210, USA
}

(Received 14 November 2005; published 26 July 2006)

\begin{abstract}
We analyze the effects of the on-site Coulomb repulsion $U$ on a band insulator using dynamical mean field theory (DMFT). We find the surprising result that the gap is suppressed to zero at a critical $U_{c 1}$ and remains zero within a metallic phase. At a larger $U_{c 2}$ there is a second transition from the metal to a Mott insulator, in which the gap increases with increasing $U$. These results are qualitatively different from Hartree-Fock theory which gives a monotonically decreasing but nonzero insulating gap for all finite $U$.
\end{abstract}

DOI: 10.1103/PhysRevLett.97.046403

PACS numbers: 71.30.+h, 71.27.+a

There are many examples of interactions opening a gap in a metallic state: the Mott metal-insulator transition, as well as spin or charge density wave gaps on nested Fermi surfaces. However, the possibility of interactions suppressing the gap in a band insulator all the way down to zero seems, at first sight, to be counterintuitive. In this Letter, we show that this does occur in a simple model treated within a well-tested approximation scheme, namely, dynamical mean field theory (DMFT) [1,2].

Specifically, we start with a simple tight-binding band insulator with two bands, one filled and the other unfilled, and turn on an on-site Coulomb repulsion, the Hubbard $U$. We treat the effect of $U$ using the DMFT within the paramagnetic sector, and show that with increasing $U$ the gap of the band insulator first collapses to zero at a $U_{c 1}$ and remains zero, leading to a metallic phase, for a finite range of $U$. This may be intuitively understood in terms of correlations screening out the one-body potential responsible for the gap in the band insulator. Eventually, with a further increase in $U$, there is a second phase transition at a $U_{c 2}$ to a Mott insulator. Our calculated phase diagram is shown in Fig. 1.

The model we consider has tight-binding electrons on a bipartite lattice (sublattices $A$ and $B$ ) described by

$$
\begin{aligned}
H= & -t \sum_{i \in A, j \in B, \sigma}\left[c_{i \sigma}^{\dagger} c_{j \sigma}+\text { H.c. }\right]+\Delta \sum_{i \in A} n_{i}-\Delta \sum_{i \in B} n_{i} \\
& +U \sum_{i} n_{i \uparrow} n_{i \downarrow}-\mu \sum_{i} n_{i},
\end{aligned}
$$

where $t$ is the nearest neighbor hopping, $U$ the Hubbard repulsion, and $\Delta$ a one-body potential which doubles the unit cell. The chemical potential is chosen [3] to be $\mu=$ $U / 2$, so that the average occupancy is $\left(\left\langle n_{A}\right\rangle+\left\langle n_{B}\right\rangle\right) / 2=1$ ("half filling"). The Hamiltonian (1) is sometimes called the "ionic Hubbard model" with $\Delta$ the "ionic" potential, and has been extensively studied especially in one dimension [4-10], although the existence of an intermediate metallic state between the band and Mott insulators is apparently not settled conclusively even in the 1D case. In any case, both our motivation and methodology are very different from previous studies.

We begin by considering various limiting cases at half filling. In the noninteracting $(U=0)$ limit the system is a band insulator with a band gap of $\Delta$. However, a very different picture emerges in the atomic limit $(t=0)$. Here we obtain a "band insulator" for $U / 2<\Delta$ with site occupancies $n_{A}=0$ and $n_{B}=2$ and a gap $\Delta-U / 2$ for either injecting or extracting an electron. This gap decreases with increasing $U$ and collapses to zero at $U / 2=$ $\Delta$ when the singly occupied states becomes degenerate with the empty one at site $A$ and with the doubly occupied one at site $B$. Beyond this point, for $U / 2>\Delta$ we get a "Mott insulator" with $n_{A}=n_{B}=1$, and a gap that goes like $U$ when $U \gg \Delta$. Hence, in this trivially soluble $t=0$ limit, we clearly see that the interaction $U$ suppresses the gap of (the state adiabatically connected to) the band insulator all the way down to zero, but only at a single

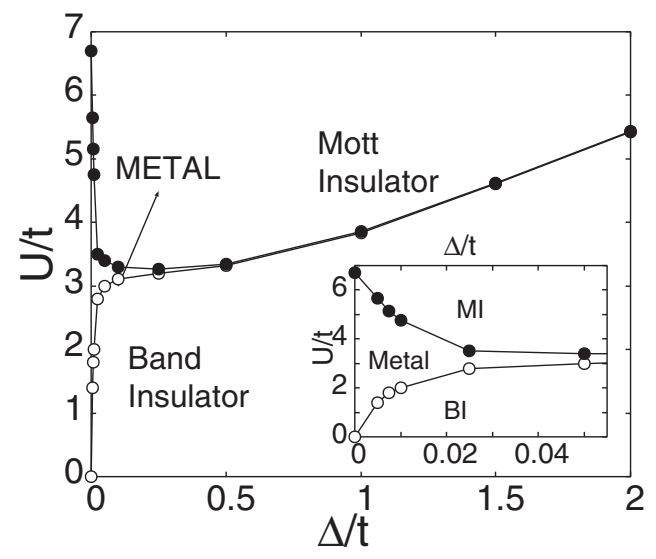

FIG. 1. The $T=0$ phase diagram of model of Eq. (1) at half filling obtained using DMFT for the Bethe lattice. The phase boundary $U_{c 1}$ is denoted by open circles while $U_{c 2}$ by filled circles. Inset: Detail of lower left-hand corner of the phase diagram. 
point $U=2 \Delta$. A question of considerable interest is whether this metallic point obtained in the atomic limit can broaden into a metallic phase when $t$ is nonzero.

Here we address this question using the DMFT approach. The DMFT approximation is exact in the limit of large dimensionality $[1,2]$ and has been demonstrated to be very successful in understanding the metal-insulator transition $[1,2]$ in the usual Hubbard model, which is the $\Delta=$ 0 limit of Eq. (1). We focus in this Letter on the paramagnetic sector of Eq. (1), for which it is convenient to introduce the matrix Green's function

$$
\hat{G}_{\alpha \beta}\left(\mathbf{k}, i \omega_{n}\right)=\left(\begin{array}{cc}
\zeta_{A}\left(\mathbf{k}, i \omega_{n}\right) & -\epsilon_{\mathbf{k}} \\
-\epsilon_{\mathbf{k}} & \zeta_{B}\left(\mathbf{k}, i \omega_{n}\right)
\end{array}\right)^{-1},
$$

where $\alpha, \beta$ are sublattice $(A, B)$ indices, $\mathbf{k}$ belongs to the first Brillouin Zone (BZ) of one sublattice, $i \omega_{n}=(2 n+$ 1) $\pi T$, and $T$ is the temperature. The kinetic energy is described by the dispersion $\epsilon_{k}$ and $\zeta_{A(B)} \equiv i \omega_{n} \mp \Delta+$ $\mu-\Sigma_{A(B)}\left(i \omega_{n}\right)$. Within the DMFT approach the selfenergy is purely local [1]. Thus the diagonal self-energies $\Sigma_{\alpha}\left(i \omega_{n}\right)$ are $\mathbf{k}$ independent and the off-diagonal selfenergies vanish (since the latter would couple the $A$ and $B$ sublattices).

We note that within the Hartree-Fock (HF) approximation the self-energy is $\Sigma_{\alpha}^{\mathrm{HF}}=U\left\langle n_{\alpha}\right\rangle / 2$ and the difference in filling factors of the two sublattices $\delta n=\left(\left\langle n_{B}\right\rangle-\left\langle n_{A}\right\rangle\right) / 2$ is given by the $T=0$ self-consistent equation $\delta n=(\Delta-$ $U \delta n / 2) \sum_{\mathbf{k}} E_{\mathbf{k}}^{-1}$, where $E_{\mathbf{k}}=\sqrt{\epsilon_{\mathbf{k}}^{2}+(\Delta-U \delta n / 2)^{2}}$ is the HF excitation spectrum. Thus, within HF theory there is a gap $(\Delta-U \delta n / 2)$ for all $\Delta \neq 0$ and the system is a band insulator for all $U$. (For $\Delta=0$, one gets $\delta n=0$ and the system is a metal for all $U$ ). However, a much richer, qualitatively different phase diagram emerges when we include fluctuations beyond HF, even at the level of the DMFT.

The DMFT approach includes local quantum fluctuations by mapping $[1,2]$ the lattice problem onto a singlesite or "impurity" with local interaction $U$ hybridizing with a self-consistently determined bath as follows. (i) We start with a guess for $\Sigma_{\alpha}\left(\omega^{+}\right)$and $\delta n$ and compute the local $G_{\alpha}\left(i \omega_{n}\right)=\sum_{\mathbf{k}} G_{\alpha \alpha}\left(\mathbf{k}, i \omega_{n}\right)$ rewritten as

$$
G_{\alpha}\left(i \omega_{n}\right)=\zeta_{\bar{\alpha}}\left(i \omega_{n}\right) \int_{-\infty}^{\infty} d \epsilon \frac{\rho_{0}(\epsilon)}{\zeta_{A}\left(i \omega_{n}\right) \zeta_{B}\left(i \omega_{n}\right)-\epsilon^{2}}
$$

with $\alpha=A(B)$ and $\bar{\alpha}=B(A)$, where $\rho_{0}(\epsilon)$ is the bare density of states (DOS) for the lattice considered (see below). We actually need to solve the problem for only one sublattice and use the relations $G_{A}\left(i \omega_{n}\right)=$ $-G_{B}\left(-i \omega_{n}\right)$ and $\Sigma_{A}\left(i \omega_{n}\right)=U-\Sigma_{B}\left(-i \omega_{n}\right)$ valid at half filling. (ii) We next determine the "host Green's function" [1,2] $\mathcal{G}_{0 \alpha}$ from the Dyson equation $\mathcal{G}_{0 \alpha}^{-1}\left(i \omega_{n}\right)=$ $G_{\alpha}^{-1}\left(i \omega_{n}\right)+\Sigma_{\alpha}\left(i \omega_{n}\right)$. (iii) We solve the impurity problem to obtain $\Sigma_{\alpha}\left(i \omega_{n}\right)=\Sigma_{\alpha}\left[G_{0 \alpha}\left(i \omega_{n}\right)\right]$. (iv) We iterate steps (i), (ii), and (iii) until a self-consistent solution is obtained.
We use as our "impurity solver" in step (iii) a generalization of the iterated perturbation theory (IPT) $[1,11]$ scheme which has the merit of giving semianalytical results directly in the real frequency $\left(\omega^{+}=\omega+i 0^{+}\right)$domain. The IPT ansatz $\Sigma_{\alpha}^{\mathrm{IPT}}\left(\omega^{+}\right)=\Sigma_{\alpha}^{\mathrm{HF}}+A_{\alpha} \Sigma_{\alpha}^{(2)}\left(\omega^{+}\right)$is constructed to be (a) exact for $U / t \ll 1$, (b) exact for $t / U=0$, and (c) exact in the large $\omega$ limit for all $U / t$, which imposes various exact sum rules. Here $\Sigma_{\alpha}^{\mathrm{HF}}$ is the HF self-energy with $n_{\alpha}=-2 \int_{-\infty}^{0} \operatorname{Im} G_{\alpha}\left(\omega^{+}\right) d \omega / \pi$, and

$$
\Sigma_{\alpha}^{(2)}\left(\omega^{+}\right)=U^{2} \int_{-\infty}^{\infty} \prod_{i=1}^{3}\left[d \epsilon_{i} \tilde{\rho}_{\alpha}\left(\epsilon_{i}\right)\right] \frac{N\left(\epsilon_{1}, \epsilon_{2}, \epsilon_{3}\right)}{\omega^{+}-\epsilon_{1}+\epsilon_{2}-\epsilon_{3}} .
$$

This has the form of the second order self-energy with $\tilde{\rho}_{\alpha}\left(\epsilon_{i}\right)=-\operatorname{Im}\left[\tilde{G}_{0 \alpha}\left(\epsilon_{i}^{+}\right)\right] / \pi, \quad$ where $\quad \tilde{\mathcal{G}}_{0 \alpha}^{-1}\left(\omega^{+}\right)=$ $G_{0 \alpha}^{-1}\left(\omega^{+}\right)-\Sigma_{\alpha}^{\mathrm{HF}}$ is the Hartree corrected host Green's function and $N\left(\epsilon_{1}, \epsilon_{2}, \epsilon_{3}\right)=f\left(\epsilon_{1}\right) f\left(-\epsilon_{2}\right) f\left(\epsilon_{3}\right)+f\left(-\epsilon_{1}\right) \times$ $f\left(\epsilon_{2}\right) f\left(-\epsilon_{3}\right)$, where $f(\boldsymbol{\epsilon})$ is the Fermi function. From condition (c) above we find that $A_{\alpha}=n_{\alpha}\left(1-n_{\alpha} / 2\right) /\left[n_{0 \alpha}(1-\right.$ $\left.\left.n_{0 \alpha} / 2\right)\right]$ with $n_{0 \alpha}=-2 \int_{-\infty}^{0} \operatorname{Im} \tilde{\mathcal{G}}_{0 \alpha}\left(\omega^{+}\right) d \omega / \pi$. Note that $A_{\alpha}$ is same for both the sublattices. The results of this simple approximate method are expected to be in semiquantitative agreement with those of more exact but numerically intensive methods $[1,2,12]$. For simplicity, here we present the results for the $T=0$ solution of DMFT equations on a Bethe lattice of connectivity $z \rightarrow \infty$. The hopping amplitude is rescaled as $t \rightarrow t / \sqrt{z}$ to get a nontrivial limit and the bare DOS is then given by $\rho_{0}(\epsilon)=$ $\sqrt{4 t^{2}-\epsilon^{2}} /\left(2 \pi t^{2}\right)$ which greatly simplifies the integral in Eq. (3). We have also solved the DMFT equations on the 2D square lattice and found that the results are qualitatively the same [13].
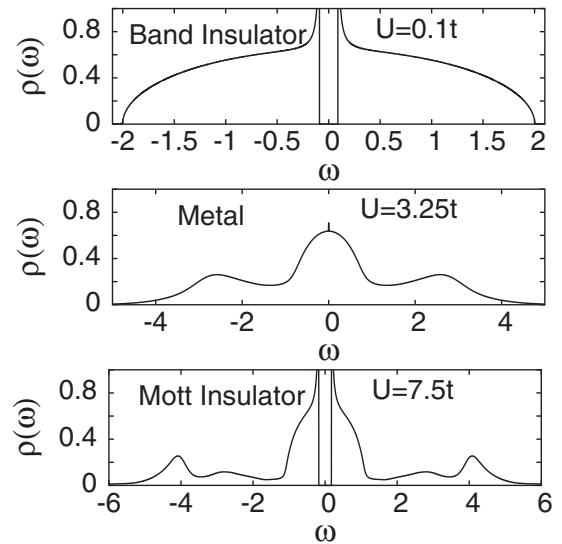

FIG. 2. The density of states $\rho(\omega)$ (in units of $1 / t$ ) plotted as a function of $\omega$ (in units of $t$ ). The three panels are all at $\Delta=0.1 t$ for various values of $U$ one for each phase. The gaps are clearly visible in the top (band insulator at $U=0.1 t$ ) and bottom (Mott insulator at $U=7.5 t$ ) panels, while the middle panel for metallic state at $U=3.25 t$ shows gapless excitations. 
The phase diagram of Fig. 1 has been obtained from the Green's function, and the (renormalized) density of states (DOS), given by $\rho(\omega)=-\sum_{\mathbf{k}} \operatorname{Im} \operatorname{Tr}\left[\hat{G}\left(\mathbf{k}, \omega^{+}\right)\right] / \pi$, calculated using the above procedure. Although the sublattice DOS $\rho_{\alpha}$ are not individually symmetric, $\rho_{B}(\omega)=$ $\rho_{A}(-\omega)$ and thus $\rho(\omega)=\rho_{A}(\omega)+\rho_{B}(\omega)$ is symmetric for half filling. Figure 2 shows how $\rho(\omega)$ evolves as a function of $U$ for a fixed $\Delta$. At small $U<U_{c 1}$ there is a gap in the spectrum, with the DOS at higher energies similar to the noninteracting (semicircular) result. We call this state a band insulator since it is adiabatically connected to the $U=0$ band insulator. At intermediate $U$ the gap collapses to zero [14], and we find a metallic state. The effects of correlations also show up in the precursors to the upper and lower Hubbard bands at higher energies. At sufficiently large $U>U_{c 2}$, the DOS again shows a gap and the system is a Mott insulator (adiabatically connected to the wellstudied Mott insulator at $\Delta=0$ ). The metallic phase, sandwiched between $U_{c 1}(\Delta)$ and $U_{c 2}(\Delta)$ in Fig. 1, shrinks as $\Delta$ increases, consistent with there being a single metallic "point" $U=2 \Delta$ in the atomic limit. We return below to the question of the scale of the critical $U_{c}$ 's as a function of $\Delta$.

The difference in filling $\delta n=\left(n_{B}-n_{A}\right) / 2$ and the energy gap are plotted in Figs. 3 and 4 as functions of $U$ for fixed $\Delta$. For $\delta n$ the DMFT results are qualitatively same as the HF results, though the $\delta n$ values within DMFT are much smaller than the HF results and become very small (of the order of error in evaluating $\delta n$ ) for large values of $U$ [15]. On the other hand, the DMFT result for the energy gap is qualitatively very different from the simple HF result (monotonically decreasing, nonzero for all $U$ ), and vanishes continuously [14] at both metalinsulator transitions, at $U_{c 1}$ and $U_{c 2}$.

Further insight into the scale of the gap and its nonmonotonic variation with $U$ is obtained by examining the self-energy $\Sigma_{\alpha}\left(\omega^{+}\right)=\Sigma_{\alpha}^{\prime}(\omega)+i \Sigma_{\alpha}^{\prime \prime}(\omega)$. From Fig. 5 we see that $\Sigma_{A}^{\prime \prime}(\omega)$ vanishes for $|\omega| \leq 3 E_{\text {gap }}$ in both the insulating phases. This can be understood from the imaginary

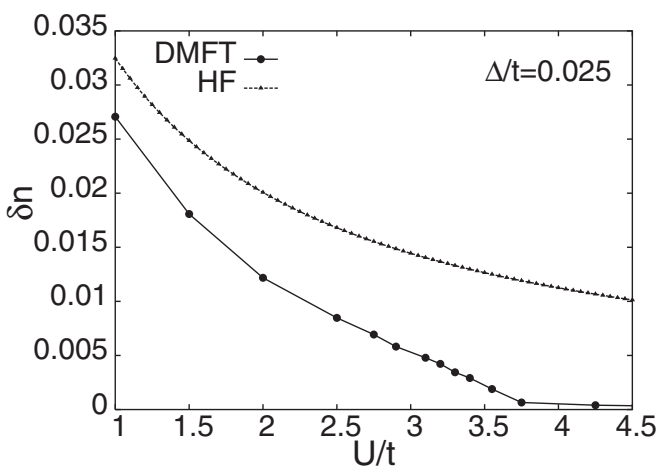

FIG. 3. Difference in filling factor $\delta n=\left(n_{B}-n_{A}\right) / 2$ for $\Delta=$ $0.025 t$ plotted as a function of $U / t$ : The DMFT result (filled circles) is much smaller than the HF result (triangles). part of $\Sigma_{\alpha}^{(2)}$ of Eq. (4), which comes from a three fermion final state. In the metallic phase $\Sigma_{A}^{\prime \prime}(\omega) \sim \omega^{2}$ near $\omega=0$, characteristic of a Landau Fermi liquid. In all the three phases, $\Sigma_{A}^{\prime}(\omega)$ can be written at low $\omega$ as a Taylor expansion $\Sigma_{\alpha}^{\prime}(\omega)=\Sigma_{\alpha}^{\prime}(0)+\left(1-Z^{-1}\right) \omega+\ldots$, where $Z$ can be shown to be independent of $\alpha$. The spectral function is defined by $\mathcal{A}_{\alpha \alpha}(\epsilon, \omega)=-(1 / \pi) \operatorname{Im} G_{\alpha \alpha}\left(\epsilon, \omega^{+}\right)$. In the insulating phases, where $\Sigma_{\alpha}^{\prime \prime}=0$ for $|\omega| \leq 3 E_{\text {gap }}$, we find from Eq. (2) that $A_{\alpha \alpha}(\epsilon, \omega)=\delta\left(r(\omega)-\epsilon^{2}\right)$ with $r(\omega)=$ $\left[\omega+\mu-\Delta-\Sigma_{A}^{\prime}(\omega)\right]\left[\omega+\mu+\Delta-\Sigma_{B}^{\prime}(\omega)\right]$. As $\epsilon$ is real, $\omega$ 's which satisfy $r(\omega)<0$ lie within the gap. The energy gap is then given by $r\left(E_{\text {gap }}\right)=0$ which, using the low-energy form of $\Sigma_{\alpha}^{\prime}$ given above and the KramersKronig relation, leads to the result

$$
E_{\text {gap }}=Z|\Delta-U \delta n / 2+S|,
$$

where $S=\mathrm{P} \int_{-\infty}^{\infty} d \omega \Sigma_{A}^{\prime \prime}(\omega) / \pi \omega$. We note that the energy gap obtained from the DOS (filled circles in Fig. 4) is in excellent agreement with that given by Eq. (5) (open squares in Fig. 4), where the error bars on the latter are obtained from the estimated numerical error in $\Sigma^{\prime \prime}$ at small $\omega$ [14]. In particular we have checked very carefully that the gap indeed vanishes in the entire metallic phase within the limits of our numerical accuracy.

We note that Eq. (5) helps greatly in clarifying several features of our results [13]. First, it shows explicitly how correlations "screen" the one-body potential $\Delta$ responsible for the gap in the band insulator and lead to a gap which is always less than the HF result in the band insulating phase, The dominant role in this being played by $S(<0)$ and by $Z(<1)$. Second, we estimate the location $U_{c 1}$ of the band insulator to metal transition using $E_{\text {gap }}=0$, which implies $U=2|\Delta+S(U)| / \delta n(U)$. We find from our numerics that although $|S(U)|$ increases with $U$, it is always much smaller than $\Delta$. Thus $U_{c 1} \simeq 2 \Delta / \delta n\left(U_{c 1}\right) \geq$ $2 \Delta / \delta n(0)$, since $\delta n(U)$ decreases with increasing $U$ (see

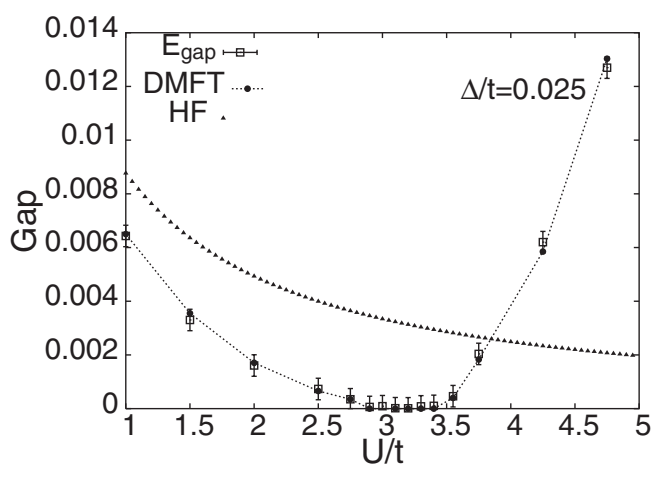

FIG. 4. The energy gap for $\Delta=0.025 t$ plotted as a function of $U / t$. The DMFT gap (dots) from the DOS and $E_{\text {gap }}$ (open squares) from Eq. (5) are in excellent agreement. Note that the gap vanishes in the metallic phase at intermediate $U$ 's. (See text for discussion of error bars). In contrast, the HF results (triangles) do not show a metallic phase. 


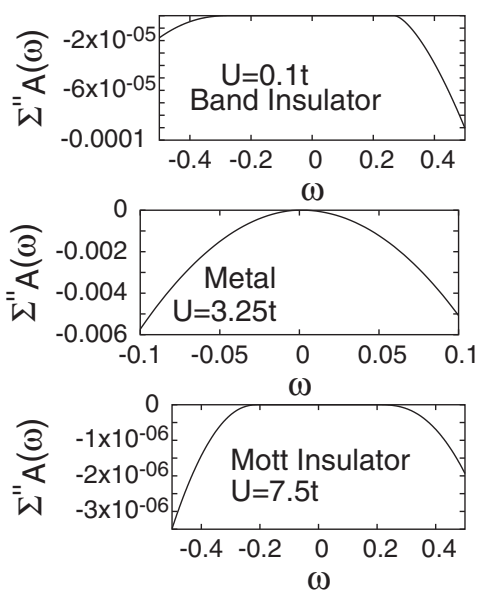

FIG. 5. Imaginary part of self-energy $\Sigma_{A}^{\prime \prime}(\omega)$ plotted as a function of $\omega$, where both are measured in units of $t$. All three panels are for $\Delta=0.1 t$ for various values of $U$. In the top (band insulator at $U=0.1 t$ ) and bottom (Mott insulator at $U=7.5 t$ ) panels $\Sigma_{A}^{\prime \prime}(\omega)$ vanishes identically for low energies $|\omega|<3 E_{\text {gap }}$. The metallic phase in the middle panel at $U=3.25 t$ has a Fermi-liquid-like $\Sigma_{A}^{\prime \prime}(\omega) \sim \omega^{2}$.

Fig. 3). Thus for small $\Delta$, we find that the very small $\delta n$ leads to $U_{c 1} \gg \Delta$; e.g., for $\Delta=0.01 t$ we get $U_{c 1} \geq$ $100 \Delta \simeq t$, while for $\Delta=0.025 t, \quad U_{c 1} \geq 20 \Delta \simeq 0.5 t$. Finally, the eventual increase in the gap with $U$ in the Mott phase, can be also traced to the $U$ dependences of $Z$ and $S$ in Eq. (5).

In the metallic phase [13] the low-energy spectral function $\mathcal{A}(\epsilon, \omega)=-\operatorname{Im} \operatorname{Tr}\left[\hat{G}\left(\epsilon, \omega^{+}\right)\right] \simeq Z \delta(\omega-Z \epsilon)$. Thus the Fermi surface is given by $\epsilon=0$, i.e., the same as for the metal with $U=\Delta=0$, as a consequence of the $\mathbf{k}$ independence of DMFT self-energy. In this phase $Z$ has the meaning of quasiparticle residue, which is also the inverse mass renormalization. Within the metallic phase, $Z$ decreases with increasing $U$ for a given $\Delta$, and with increasing $\Delta$ for fixed $U$.

In conclusion, we have analyzed a simple model of a band insulator with on-site Coulomb repulsion $U$. At the Hartree-Fock level the gap is reduced but the insulating behavior persists. However, when we treat correlation effects using DMFT we find the surprising result that the gap is suppressed to zero and there is a band insulator to metal transition at a critical $U_{c 1}$. At a larger $U_{c 2}$ there is a second transition from the metal to a Mott insulator.

Our work raises several important questions. First, our results are obtained in the paramagnetic sector. We expect that the metallic phase will survive if antiferromagnetism [13] is suppressed due to frustration, in much the same way as in the DMFT treatment in the $\Delta=0$ limit [1]. It would be interesting to treat a model in which such frustration is explicitly included. Second, it is important to ask if in finite dimensions, i.e., with $\mathbf{k}$-dependent self-energies, other broken symmetry states might also appear, for example, the bond ordered state proposed as an intermediate phase in low dimensional studies [8-10]. Finally, it would be most interesting to look for experimental systems, either transition metal oxides [16] or fermions in optical lattices [17], where increasing correlations could drive a band-insulator metallic. All these are questions for future work.

M. R. would like to thank N. Trivedi for very useful conversations.

[1] A. Georges, G. Kotliar, W. Krauth, and M. J. Rozenberg, Rev. Mod. Phys. 68, 13 (1996).

[2] T. Pruschke, M. Jarrell, and J. K. Freericks, Adv. Phys. 44, 187 (1995).

[3] This can be shown by using particle-hole symmetry at half filling.

[4] J. Hubbard and J. B. Torrance, Phys. Rev. Lett. 47, 1750 (1981).

[5] T. Egami, S. Ishihara, and M. Tachiki, Science 261, 1307 (1993).

[6] G. Ortiz and R. Martin, Phys. Rev. B 49, 14202 (1994).

[7] R. Resta and S. Sorella, Phys. Rev. Lett. 74, 4738 (1995); 82, 370 (1999).

[8] M. Fabrizio, A. O. Gogolin, and A. A. Nersesyan, Phys. Rev. Lett. 83, 2014 (1999).

[9] T. Wilkens and R. M. Martin, Phys. Rev. B 63, 235108 (2001).

[10] C. D. Batista and A. A. Aligia, Phys. Rev. Lett. 92, 246405 (2004).

[11] H. Kajueter and G. Kotliar, Phys. Rev. Lett. 77, 131 (1996).

[12] R. Bulla, T. A. Costi, and D. Vollhardt, Phys. Rev. B64, 045103 (2001).

[13] Further details will be published elsewhere; A. Garg, H. R. Krishnamurthy, and M. Randeria (unpublished).

[14] The very low-energy results for the DOS and $\Sigma^{\prime \prime}$ are highly susceptible to numerical errors, particularly in the metallic phase. We have checked that our results are robust under increased numerical accuracy. In many cases we have also compared results using two different techniques (fast Fourier transform and direct numerical integration). This forms the basis for error bars on $E_{\text {gap }}$ in Fig. 4. Within our numerical accuracy, we have found no evidence so far for coexisting phases or hysteretic behavior for nonzero $\Delta$.

[15] For $U \gg t, \Delta$, the leading contribution to the energy of model (1), comes from a Heisenberg term with superexchange $J=4 t^{2} U /\left(U^{2}-4 \Delta^{2}\right)$ and we can show that $\delta n \sim t^{2} \Delta / U^{3}$. Although the $\delta n$ calculated using DMFT + IPT need not agree with this exact asymptotic form in detail, we nevertheless expect that it is nonzero for large $U / t$ values, where we cannot reliably estimate $\delta n$ from our numerics.

[16] M. Imada, A. Fujimori, and Y. Tokura, Rev. Mod. Phys. 70, 1039 (1998).

[17] M. Kohl, H. Moritz, T. Stoferle, K. Gunter, and T. Esslinger, Phys. Rev. Lett. 94, 080403 (2005). 